\title{
Is Fast Track protocol a safe tool to reduce hospitalization time after radical cystectomy with ileal urinary diversion? Initial results from a single high-volume centre
}

\author{
Daniele Romagnoli ${ }^{1}$, Riccardo Schiavina ${ }^{2}$, Lorenzo Bianchi ${ }^{2}$, Marco Borghesi ${ }^{2}$, Francesco Chessa ${ }^{2}$, \\ Federico Mineo Bianchi ${ }^{2}$, Andrea Angiolini ${ }^{2}$, Carlo Casablanca ${ }^{2}$, Marco Giampaoli ${ }^{1}$, Paolo Corsi ${ }^{1}$, \\ Daniele D'Agostino $^{1}$, Eugenio Brunocilla ${ }^{2}$, Angelo Porreca ${ }^{1}$ \\ ${ }^{1}$ Abano Terme Hospital, Abano Terme (PD), Italy; \\ ${ }^{2}$ St. Orsola Malpighi Universitary Urology Clinic, Bologna (BO), Italy.
}

\begin{abstract}
Summary Introduction and aim: Radical Cystectomy (RC) with ileal urinary diversion is one of the most complex urological surgical procedure, and many Fast Track (FT) protocols have been described to reduce hospitalization, without increasing postoperatory complications. We present the one-year results of a dedicated protocol developed at a high volume centre.

Materials and methods: The FT protocol was designed after a review of the literature and a multidisciplinary collegiate discussion, and it was applied to patients scheduled to open RC with intestinal urinary diversion. To validate its feasibility, we compared its results with data collected from a 1:1 matched population of patients who had undergone the same surgical procedure, without the implementation of the FT protocol. Results: We enrolled in the FT group 11 (55\%) patients scheduled to RC with ileal conduit diversion, and 9 patients (45\%) scheduled to orthotopic neobladder (Studer) substitution, while a numerically equivalent population was enrolled in the control group, matched according to age at surgery, BMI, gender, ASA score, CCI, preoperative stage and type of urinary diversion. No statistically significant difference was found in terms of pre-operatory and intra-operatory domains. Median overall age was 71 years (Inter Quartile Range - IQR: 63-76) and mean operatory time was $276 \pm 57$ minutes. Hospitalization time was significantly reduced in the FT group, considering oralization and canalization items we found a significant advantage in the FT group. No statistically significant difference was found in the control of the post-operatory pain. We found no difference, in terms of both early and late complications ratio, among the two populations. Complications graded Clavien $\geq 3$ were found in 4 patients of the control group (20\%), while in only one patient (5\%) in the Fast Track group, though this difference was not statistically significant.

Conclusions: The Fast Track protocol developed in this study has proven to be effective in significantly reducing hospitalization time in patients submitted to $R C$ with intestinal urinary diversion, without increasing post-operatory complications ratio.
\end{abstract}

KEY WORDS: Radical cystectomy; Fast Track; Enhanced recovery; After surgery.

Submitted 4 June 2019; Accepted 26 June 2019

\section{INTRODUCTION}

Bladder cancer (BC) represents the $7^{\text {th }}$ most common cancer in male population and the $11^{\text {th }}$ considering both sexes (1). Open RC remains the gold standard for the surgical treatment of localized muscle invasive bladder cancer (MIBC) or non-muscle invasive bladder cancer (NMIBC) resistant to topic chemo- and immunologic therapy $(2,3)$. RC with urinary diversion is considered one of the most complex urological surgery and is characterized by long hospital stay and high rate of postoperative morbidity and mortality. Complication rate could be up to $34.4 \%$ (and to $50 \%$ in some series) for mildmoderate grade (Clavien Dindo < 3), and up to $17.5 \%$ for severe grade $(4,5)$ (Clavien Dindo $\geq 3$ ). Even if improvements in surgical procedure have reduced incidence of postoperative complication, it remains important to minimise surgical trauma and optimise perioperative care.

The term "Fast Track" refers to a group of perioperative protocols aimed to standardise perioperative cares, shorten hospital stay, maintain optimal surgical treatment quality without increasing postoperative complication rate (6). They are also commonly known as Enhanced Recovery After Surgery (ERAS) protocols, as they were firstly described in general surgery. FT schemes are standardised, multimodal and multidisciplinary developed protocols aimed to enhance surgical outcomes referring to perioperative "best clinical practice" (7). The origins of ERAS protocols date back to the early 90s with the experiences of Dahl et al., with bupivacaine intratecal analgesia (8), and Kehlet et al. with epidural anaesthesia, high preoperative glucose intake and early mobilization and starting of oral diet, applied on colorectal surgery with a mean reduction of 2 days in terms of hospital stay (9). From the urologist's point of view, FT protocols can be applied mainly to RC with ileal diversion, considering the complex operation technique, high complication rate and long mean hospital stay.

Distinctive tract of the FT protocols is that they can be adapted on patient's needs depending on perioperative management phase. Key features of FT protocols are: perioperative diet management, advanced anesthesiological technique, specific antalgic postoperative care (based on non-opioid drugs), early oral diet intake and mobilization (10). We developed a FT protocol with the aim of reducing mean hospitalization time in patients sub- 
mitted to RC with ileal urinary diversion in our centre, without increasing the complications ratio. histological tumor features (such as stage, grading, lymph node status), hospital stay time, NGT removal

\section{Materials AND METHOdS}

After an extensive review of literature and a multidisciplinary team consult consisting of urologists, anesthesiologists, nurses and nutritionists, we developed an ERAS protocol (see Appendix). To test the effect of this protocol, we designed a pilot observational prospective cohort study, in accordance with the principles and practice of our Review Board. The protocol focused on the reduction of postoperative nausea and vomiting, early canalization, nasogastric tube (NGT) removal, enteral feeding and mobilization, shorter hospitalization time, without significant worsening in terms of complication rate or pain management.

We enrolled 20 consecutive patients candidate to open RC with ileal urinary diversion from January 2016 to April 2017 at a single high volume centre. Each operation was performed by surgeons at the end of the learning curve and with extensive experience. The indications for RC included muscle-invasive bladder carcinoma or high-grade non-muscle invasive bladder carcinoma refractory to topic intravesical immunotherapy in fit-for-surgery patients $(2,3)$. Preoperative radiological assessment was realized via a toraco-abdominal computed tomography with urographic reconstructions and contrast enhanced magnetic resonance of the pelvis (we adopted this accessory technique in order to have a precise and detailed study of the pelvis, as previously described) (11). Data were prospectively collected from medical records. For each patient of the study population a one-to-one propensity scorematched analysis was performed with a population selected among 64 patients who underwent RC with ileal urinary diversion, without application of the FT protocol. Each patient received detailed instructions about FT proto$\mathrm{col}$ at preoperative evaluation. Adherence to instructions was verified at the time of the hospital admittance. Data were prospectively collected for patients in the FT group, while, for patients of the control group, each item was retrospectively collected.

Preoperative data were collected about age, Body Mass Index (BMI), American Society of Anesthesiology score (ASA Score), Charlson Comorbidity Index (CCI), smoking habits, clinical stage, grading (defined sec. WHO 2016 classification) or neoadiuvant therapy. We collected data regarding surgical approach, urinary diversion used, pelvic lymphadenectomy template, number of removed lymph nodes, global operation time (minutes) and intraoperative transfusion rate.

Postoperative data collection comprehended

Table 1.

Preoperative and intraoperative items.

\begin{tabular}{|c|c|c|c|c|}
\hline & Overall & Fast Track group & Control group & $P$ value \\
\hline Number of patients, $n(\%)$ & $40(100 \%)$ & $20(50 \%)$ & $20(50 \%)$ & - \\
\hline \multicolumn{5}{|l|}{ Gender, n (\%) } \\
\hline Male & $31(77.5)$ & $16(80)$ & $15(75)$ & 0.7 \\
\hline Female & $49(22.5)$ & $4(20)$ & $5(25)$ & \\
\hline \multicolumn{5}{|l|}{ Age } \\
\hline Median (IQR) & $71(63-76)$ & $70(60-76)$ & $72(66-75)$ & 0.6 \\
\hline \multicolumn{5}{|l|}{ ASA score, $n(\%)$} \\
\hline $1-2$ & $16(40)$ & $9(45)$ & $7(35)$ & 0.5 \\
\hline $3-4$ & $24(60)$ & $11(55)$ & $13(65)$ & \\
\hline \multicolumn{5}{|l|}{$\overline{\mathrm{CCl}, \mathrm{n}(\%)}$} \\
\hline 0 & $0(0)$ & $0(0)$ & $0(0)$ & 0.8 \\
\hline $1-2$ & $7(17.5)$ & $4(20)$ & $3(15)$ & \\
\hline 3-4 & $15(37.5)$ & $8(40)$ & $7(35)$ & \\
\hline$>4$ & $18(45)$ & $8(40)$ & $10(50)$ & \\
\hline \multicolumn{5}{|l|}{$\overline{B M I}\left(\mathrm{Kg} / \mathrm{m}^{2}\right)$} \\
\hline Mean \pm SD & $28 \pm 4.8$ & $28 \pm 4.9$ & $28 \pm 5$ & 0.9 \\
\hline \multicolumn{5}{|l|}{ Smoking attitude, n (\%) } \\
\hline No & $16(40)$ & $11(55)$ & $5(25)$ & 0.05 \\
\hline Yes & $24(60)$ & $9(45)$ & $15(75)$ & \\
\hline \multicolumn{5}{|l|}{ Clinical stage, n (\%) } \\
\hline TO & $1(2.5)$ & $0(0)$ & $1(5)$ & 0.7 \\
\hline Ta-pT1 & $12(30)$ & $7(35)$ & $5(25)$ & \\
\hline T2 & $23(57.5)$ & $11(55)$ & $12(60)$ & \\
\hline $\mathrm{T} 3$ & $4(10)$ & $2(10)$ & $2(10)$ & \\
\hline \multicolumn{5}{|l|}{ Preoperative grade, $n(\%)$} \\
\hline G1 & $1(2.5)$ & $0(0)$ & $1(5)$ & 0.6 \\
\hline G2 & $5(5)$ & $1(5)$ & $1(5)$ & \\
\hline G3 & $37(92.5)$ & $19(95)$ & $18(90)$ & \\
\hline \multicolumn{5}{|l|}{ Neoadjuvant chemotherapy, n (\%) } \\
\hline No & $40(100)$ & $40(100)$ & $40(100)$ & - \\
\hline Yes & $0(0)$ & $0(0)$ & $0(0)$ & \\
\hline \multicolumn{5}{|l|}{ Surgical approach, $n(\%)$} \\
\hline Open & $39(97.5)$ & $19(95)$ & $20(100)$ & 0.3 \\
\hline Laparoscopic & $1(2.5)$ & $1(5)$ & $0(0)$ & \\
\hline \multicolumn{5}{|l|}{ PLND template, $n(\%)$} \\
\hline Not performed & $2(5)$ & $1(5)$ & $1(5)$ & 0.2 \\
\hline Standard & $22(55)$ & $13(65)$ & $9(45)$ & \\
\hline Extended & $14(35)$ & $4(20)$ & $10(50)$ & \\
\hline Super-extended & $2(5)$ & $2(10)$ & $0(0)$ & \\
\hline \multicolumn{5}{|l|}{ Lymph node retrieved } \\
\hline Median (IQR) & $14(10-23)$ & $14(12-21)$ & $14(8-24)$ & 0.3 \\
\hline \multicolumn{5}{|l|}{ Urinary diversion, $\mathrm{n}(\%)$} \\
\hline lleal conduit & $23(57.5)$ & $11(55)$ & $12(60)$ & 0.7 \\
\hline Ileal ortotopic neobladder (Studer neobladder) & $17(42.5)$ & $9(45)$ & $8(40)$ & \\
\hline \multicolumn{5}{|l|}{ Surgical time (minutes) } \\
\hline Mean \pm SD & $276 \pm 57$ & $260 \pm 56$ & $293 \pm 54$ & 0.06 \\
\hline \multicolumn{5}{|l|}{ Intraoperative transfusion, n (\%) } \\
\hline No & $27(67.5)$ & $15(75)$ & $12(60)$ & 0.3 \\
\hline Yes & 13 (32.5) & $5(25)$ & $8(40)$ & \\
\hline Pathologic stage, $n$ (\%) & & & & \\
\hline pTO & $2(5)$ & $1(5)$ & $1(5)$ & 0.8 \\
\hline pT1-pTis & $9(22.5)$ & $6(30)$ & $3(15)$ & \\
\hline рт2a-рT2b & $7(17.5)$ & $3(15)$ & $4(20)$ & \\
\hline рТЗа-рТЗь & $15(37.5)$ & $6(30)$ & $9(45)$ & \\
\hline pT4 & $7(17.5)$ & $4(20)$ & $3(15)$ & \\
\hline Pathologic grade, $n(\%)$ & & & & \\
\hline G1 & $2(5)$ & $1(5)$ & $1(5)$ & 0.8 \\
\hline G2 & $2(5)$ & $1(5)$ & $1(5)$ & \\
\hline G3 & $35(87.5)$ & $17(85)$ & $18(90)$ & \\
\hline G4 & $1(2.5)$ & $1(5)$ & $0(0)$ & \\
\hline$\overline{\mathrm{LN}}, \mathrm{n}(\%)$ & & & & \\
\hline No & $26(65)$ & $13(65)$ & $13(65)$ & 1 \\
\hline Yes & $14(35)$ & $7(35)$ & $7(35)$ & \\
\hline
\end{tabular}


(time and repositioning), pain control (coded by visual analogic scale [VAS] standard), time to flatus and time to defecation, lymphorrea amount, time of the start of a light diet and time to drain removal. Postoperative complications were stratified as early (before 30 days from surgery) and late complications (between 30 and 90 days from surgery).

All complications were graded following the Clavien-Dindo classication.

To compare results between the study population and the control group a one-to-one propensity score-matched analysis was computed by modelling a logistic regression, with the dependent variable as the odds of undergoing Fast Track protocol and independent variables such as age at surgery, BMI, gender, ASA score, CCI, preoperative stage and urinary diversion in course of surgery.

Subsequently, covariate balance between the matched groups was examined. Covariates between the two groups were considered equivalent, providing a standardised mean difference $\leq 10 \%$. The primary objective was the evaluation of the eventual reduction in hospitalization ratio, while the secondary objective was the evaluation of any difference in the early ( $<30$ days) and late (< 90 days) postoperative complication ratio. Statistic software $\mathrm{R}$ (The $R$ Foundation) was used for statistical analysis. Chi-square test and t test were used for binomial and continuous variables, respectively.

\section{RESULTS}

11 (55\%) patients of FT groups underwent ureteroileocutaneostomy, while 9 (45\%) patients underwent urinary diversion with orthotopic neobladder (according to the Studer technique), while, in the control group, 12 (60\%) patients received ureteroileocutaneostomy and 8 patients (40\%) were submitted to orthotopic urinary diversion according to the Studer technique. Table 1 shows preoperative and intraoperative characteristics of the two study groups. The two groups were statistically homogenous, with no significant difference among them. Considering intraoperative parameters, mean operative time was $260 \pm 56$ min in FT group, while was $293 \pm 54$ min in the control group, with a difference at the limit of the statistical difference ( $p=0.06)$.

Table 2 depicts Fast Track outcomes. NGT was removed earlier in the FT group than in the control group, with a median of 20 hours versus 48 hours, respectively ( $p<0.001$ ). Just one patient (5\%) of the FT group had SNG removed after the first 24 hours. No significant difference was noted in NGT repositioning rates between the two groups (15\% in FT group and $5 \%$ in control group, $\mathrm{p}=0.3)$.
Table 2.

Postoperative datas.

\begin{tabular}{|c|c|c|c|c|}
\hline & Overall & Fast Track group & Control group & $P$ value \\
\hline Number of patients, $n$ (\%) & $40(100 \%)$ & $20(50 \%)$ & $20(50 \%)$ & - \\
\hline \multicolumn{5}{|l|}{ NGT removal ( hours) } \\
\hline Median (IQR) & $24(20-48)$ & $20(18-20)$ & $48(48-72)$ & $<0.001$ \\
\hline$\overline{\text { NGT removal after POD 1, n (\%) }}$ & $18(42.5)$ & $1(5)$ & $16(80)$ & $<0.001$ \\
\hline NGT repositioning, n (\%) & $4(10)$ & $3(15)$ & 1 (5) & 0.3 \\
\hline \multicolumn{5}{|l|}{ Mobilization (hours postoperatively) } \\
\hline Mean $\pm S D$ & $66 \pm 43$ & $21 \pm 12$ & $106 \pm 26$ & $<0.001$ \\
\hline \multicolumn{5}{|l|}{ Postoperative nausea episodes, n (\%) } \\
\hline None & $25(62.5)$ & $13(65)$ & $12(60)$ & 0.02 \\
\hline 1 & $7(17.5)$ & $6(30)$ & $1(5)$ & \\
\hline$>1$ & $8(20)$ & $1(5)$ & $7(35)$ & \\
\hline \multicolumn{5}{|l|}{ Postoperative VAS Score } \\
\hline Median (IQR) & $4(3-4)$ & $3(3-4)$ & $4(3-4)$ & 0.2 \\
\hline \multicolumn{5}{|l|}{ Time to flatus (POD) } \\
\hline Median (IQR) & $2(1-3)$ & $1.5(1-2.75)$ & $3(2-3.75)$ & 0.004 \\
\hline \multicolumn{5}{|l|}{ Time to defecation (POD) } \\
\hline Median (IQR) & $5(3-6)$ & $4(3-5.75)$ & $6(4.5-6)$ & 0.02 \\
\hline \multicolumn{5}{|l|}{ Start of a light diet (POD) } \\
\hline Median (IQR) & $5(2-8)$ & $2(2-4.5)$ & $6.5(6-8)$ & $<0.001$ \\
\hline \multicolumn{5}{|l|}{ Last drain removal (POD) } \\
\hline Median (IQR) & $8(6-9)$ & $7(6-9.75)$ & $8.3(7-9)$ & 0.5 \\
\hline \multicolumn{5}{|l|}{$\begin{array}{l}\text { Lymphorrea amount (ml) } \\
\text { (m) }\end{array}$} \\
\hline Mean \pm SD & $1720 \pm 1534$ & $1776 \pm 1710$ & $1665 \pm 1380$ & 0.8 \\
\hline \multicolumn{5}{|l|}{ Hospital stay (days) } \\
\hline Median (IQR) & $12(9-14)$ & $10(8-12)$ & $13(11-14)$ & 0.005 \\
\hline
\end{tabular}

Table 3.

Postoperative complications.

\begin{tabular}{|c|c|c|c|c|}
\hline & Overall & Fast Track group & Control group & $P$ value \\
\hline Number of patients, $n(\%)$ & $40(100 \%)$ & $20(50 \%)$ & $20(50 \%)$ & - \\
\hline \multicolumn{5}{|c|}{ Overall perioperative (<30 days) complications, $n(\%)$} \\
\hline No & $31(77.5)$ & $14(70)$ & $17(85)$ & 0.3 \\
\hline Yes & $9(22.5)$ & $6(30)$ & $3(15)$ & \\
\hline \multicolumn{5}{|l|}{ Clavien-Dindo classification, $n$ (\%) } \\
\hline Grade 1 & $6(66.6)$ & $5(83.3)$ & $1(33.3)$ & 0.3 \\
\hline Grade 2 & $2(22.2)$ & $1(16.7)$ & $1(33.3)$ & \\
\hline Grade 3 & $1(11.1)$ & $0(0)$ & $1(33.3)$ & \\
\hline Grade 4 & $0(0)$ & $0(0)$ & $0(0)$ & \\
\hline Grade 5 & $0(0)$ & $0(0)$ & $0(0)$ & \\
\hline \multicolumn{5}{|c|}{ Type of complication (<30 days), $n$ (\%) } \\
\hline Dynamic Ileus & $6(66.6)$ & $5(83.3)$ & $1(33.3)$ & 0.3 \\
\hline Anemization & $1(11.1)$ & $1(16.7)$ & $0(0)$ & \\
\hline Wound Infection & $1(11.1)$ & $0(0)$ & $1(33.3)$ & \\
\hline Deep Venous Thrombosis & $1(11.1)$ & $0(0)$ & $1(33.3)$ & \\
\hline \multicolumn{5}{|c|}{ Overall postoperative (< 90 days) complications, $n(\%)$} \\
\hline No & $36(90)$ & $19(95)$ & $17(85)$ & 0.3 \\
\hline Yes & $4(10)$ & $1(5)$ & $3(15)$ & \\
\hline \multicolumn{5}{|l|}{ Clavien-Dindo classification, $n$ (\%) } \\
\hline Grade 1 & $0(0)$ & $0(0)$ & $0(0)$ & 0.3 \\
\hline Grade 2 & $0(0)$ & $0(0)$ & $0(0)$ & \\
\hline Grade 3 & $4(100)$ & $1(100)$ & $2(100)$ & \\
\hline Grade 4 & $0(0)$ & $0(0)$ & $0(0)$ & \\
\hline Grade 5 & $0(0)$ & $0(0)$ & $0(0)$ & \\
\hline \multicolumn{5}{|c|}{ Type of complication (<90 days), $n(\%)$} \\
\hline Lymphocele (Right lliac Fossa) & $1(25)$ & $1(100)$ & 0 & 0.2 \\
\hline Uretero-lleal Anastomosis Stricture & $2(50)$ & 0 & $2(66.7)$ & \\
\hline Laparocele & $1(25)$ & 0 & $1(33.3)$ & \\
\hline Readmission within 90 days, $\mathrm{n}(\%)$ & $4(10)$ & $1(5)$ & $3(15)$ & 0.3 \\
\hline
\end{tabular}


Mobilization time was significantly shorter in the FT group than in the control group (mean $21 \pm 12$ hours vs $106 \pm 26$ hours, respectively, $\mathrm{p}<0.001$ ). We observed one (5\%) patient in the FT group who had more than one nausea episode postoperatively, while we noted 7 (35\%) of such events in the control group $(\mathrm{p}=0.02)$. Median time to flatus was 1,5 (IQR 1-3) days in the FT group and 3 (IQR: 2-3.75) days in the control group, with a statistically significant difference $(\mathrm{p}=0.004)$. We also observed a significant shorter time to defecation in the FT group (4 days IQR: $3-5.75$ ) than in the control group (6 days, IQR 4.5-6) with $\mathrm{p}=0.02$. No statistically significant difference was noted in terms of VAS scale, duration and entity of lymphorrea between the two groups. We observed a statistically significant shorter median hospital stay time in the FT group (10 days, IQR: 8-12) than in the control group (13 days, IQR: 11-14, $\mathrm{p}=0.005$ ).

As depicted in Table 3, no statistically significant difference was noted in terms of early and late complication rate among the two groups ( $\mathrm{p}=0.3$ for each type of complication, respectively). Considering early complications, only one event graded as Clavien 3 was reported (11.1\%), and it was a wound infection surgically treated in the control group. Considering late complications ratio, 4 events graded as Clavien 3 were documented, 1 in the FT group (right iliac fossa lymphocele percutaneously drained), and 3 in the control group ( 2 cases of uretero-ileal anastomosis stricture surgically corrected, and 1 case of laparocele surgically repaired), though this difference was not statistically significant $(p=0.2)$. No statistically significant difference was found in terms of readmission rate within 90 days among the two groups $(p=0.3$ )

\section{Discussion}

RC with ileal urinary diversion is a surgery historically affected by a high rate of perioperative morbidity and mortality. With the starting point set in intervention on bowel in general surgery, ERAS protocols were described in order to improve postoperative outcomes. Although a number of ERAS protocols have been built over the years, all of them found their key features on strategies to improve postoperative recovery rate and reduction of hospital stay time, without worsening postoperative complication rate. After extensive literature review and multidisciplinary meeting between urologists, anaesthetists, nurses and nutritionists, we designed a tailored ERAS protocol to be adopted at a high volume institution. In order to validate the FT protocol we designed a case-control prospective study, matching patients who underwent RC with ileal urinary diversion and who applied the protocol with patients who underwent the same kind of surgery but without implementation of the protocol. In our cohorts of RC with ileal urinary diversion, the adherence to the FT protocol permitted to obtain a significant shorter hospitalization time, without a significant increase in term of perioperative complications rate. An interesting fact is that no preoperative bowel preparation was adopted, because, as demonstrated by Shafii et al., it does not give any significant advantage (12). Moreover, the early removal of the NGT tube, in adjunction with a continuous prokinetic stimu- lus, has proven to be feasible, in accordance to the experience of Braga (13), who demonstrated that decompression with NGT in all patients is not necessary ad is associated with an increased incidence of pulmonary complications. An important contribute to this result is represented by the perioperative dietary regimen and by the intra- and postoperative pain management. The hypercaloric and hyperglucidic preoperative dietary regimen of the FT protocol allows to create a preoperative supply of proteins and glucose in order to react to the operative stress without significantly compromise the homeostasis and improving the natural healing process. This fact seems to be the possible base for the observation that no wound infections were reported in the FT group. As a matter of fact, wound repair depends on the disponibility of adequate protein and glucose supply, which could be insufficient after a prolonged perioperative fasting period.

We observed no statistically significant difference in VAS scale evaluation between the two groups, so we might affirm the non-inferiority of an opioid-free pain control regimen (based on FANS and continuous infusion via epidural catheter), in comparison with the pain control obtained with opioid drugs. Moreover, the absence of opioid administration allows to avoid typical side effects, such as a prolonged intestinal transit, which could hesitate in delayed time to flatus and time to defecation. Other aspects of our FT protocol aimed to improve intestinal function, such as administration of prokinetic drugs (metoclopramide) and of chewing-gum, as already been prove successful by Kouba et al. (14). We observed a statistically significant reduction of canalization time in FT group compared to control group, either considering median time to flatus (respectively in POD 1,5 vs POD $3, p=0.004$ ) and median time to defecation (respectively on POD 4 vs POD 6, p = 0.02). Moreover we observed that patients of the FT group could tolerate a solid diet regimen on $\mathrm{POD} 2$, significantly sooner in comparison with patients of the control group (median POD 6.5, $\mathrm{p}<$ 0.001 ). These results could be explained by the fact that metoclopramide administration is able to reduce the incidence of nausea and vomiting episodes, and also gastrointestinal complications, as described by Pruthi (15). Another explanation for this matter could be the fact that faster bowel activity recovery might be reached also with early mobilization and early feeding, as postulated by Cerruto et al. (10). Internal peristalsis is moreover facilitated by the blocking of visceral afferents and segmental efferences, which is realized by the epidural analgesia (16). The importance of a T11 epidural catheter as a useful tool to increase microvessels perfusion (thus reducing interference with the cardiopulmonary system), has been underlined by Friedrich-Freksa, who successfully applied this technique to high-risk patients submitted to RC (17).

The result of the aforementioned considerations allows patients in the FT group to be discharged 3 days before, in comparison with patients of the control group (mean 10 days vs 13 days, $\mathrm{p}=0.005$ ). This result is in line with the Literature, though there are discordant experiences, as the one described by Cerruto (10), who reported no statistically significant difference in mean hospital stay 
time in patients submitted to RC with implementation of a dedicated FT protocol.

A promising synergy is represented by FT protocols applied to mini-invasive surgery, a technique which is usually already characterized by a short hospital stay (18). As demonstrated by Saar et al. (19), the implementation of a FT protocol in case of robotic approach to $\mathrm{RC}$ provides a significant advantage in terms of return to a regular diet and use of postoperative morphine equivalents.

The efficacy of FT protocols applied to mini-invasive surgery has led to the recommendation to always adopt them in case of robot assisted radical cystectomy, as written in the paper published by Wilson (20). Moreover, the use of barbed sutures (21), a typical feature of the robotic approach, might improve the postoperatory continence ratio, as described in case of robotassisted radical prostatectomy $(22,23)$.

Another point of interest of FT protocols is the eventual reduction of both postoperative complications ratio and 90 -days readmission rates. On a previous publication by Cerruto et al. on the effect of a FT protocol in patients candidates to robot-assisted RC with Padovana ileal neobladder, the authors reported a lower global rate of postoperative complications $(\mathrm{p}=0.004)$ in patients adherent to the protocol (10).

In our study we did not record a statistically significant difference between the two groups in terms of complications or readmission rate. Such observation is in partly due to the scarce numerosity of the group of our study. It is important to underline that no major early complication (grade 3 or superior according to the Clavien-Dindo classification) was observed in the FT group. Moreover, of the 4 (10\%) major late complications observed, only 1 ( $5 \%$ ) was in FT group, while 3 (15\%) were in control group.

The complication of the FT group was a lymphocele treated with ultrasound-guided percutaneous drainage, which seemed unrelated to FT protocol implementation. On the control group we observed one early and three late complications Clavien $\geq 3$, consisting of wound infection (early complication), two uretero-ileal anastomotic strictures (a well-documented complication of this kind of surgery) (24) and a laparocele, all of them treated with surgical revision on general anaesthesia.

A limitation of the present study is the limited number of patients enrolled, though the scarce numerosity seems to be a common feature in studies concerning FT protocols applied to RC, as confirmed by a recent paper published by Freeks et al. (25).

\section{Conclusions}

The implementation of the FT protocol to patients submitted to RC with urinary ileal diversion is a safe and effective procedure, which allows to reduce hospitalization time without increasing postoperatory complications ratio. Further studies are needed, with larger populations, in order to definitively confirm the superiority of FT protocols over standard protocols in the perioperative management of patients submitted to this surgical procedure.

\section{References}

1. Ferlay J, et al. Cancer incidence and mortality patterns in Europe: Estimates for 40 countries in 2012. Eur J Cancer. 2013; 49:13741403.

2. Babijuk M, et al. EAU Guidelines: Non-muscle-invasive Bladder Cancer, 2019.

3. Witjes JA, et al. EAU Guidelines: Muscle-invasive and Metastatic Bladder Cancer, 2019.

4. Schiavina $R$, et al. Perioperative Complications and Mortality After Radical Cystectomy When Using a Standardized Reporting Methodology. Clin Genitourin Cancer. 2013; 11:189-197.

5. Chung Y-R, et al. Association of statin use and hypertriglyceridemia with diabetic macular edema in patients with type 2 diabetes and diabetic retinopathy. Cardiovasc Diabetol. 2017; 16:4.

6. Geltzeiler CB, et al. Prospective Study of Colorectal Enhanced Recovery After Surgery in a Community Hospital. JAMA Surg. 2014; 149:955-961.

7. Chang SS, et al. Analysis of Early Complications After Radical Cystectomy: Results of a Collaborative Care Pathway, J Urol. 2002; 167:2012-6.

8. Dahl JB, et al. Influence of timing on the effect of continuos extradural analgesia with bupivacaine and morphine after major abdominal surgery, Br J Anaesth. 1992; 69:4-8.

9. Kehlet $H$, et al. Multimodal strategies to improve surgical outcome. Am J Surg. 2002; 183:630-641.

10. Cerruto MA, et al. Fast track surgery to reduce short-term complications following radical cystectomy and intestinal urinary diversion with Vescica Ileale Padovana neobladder: proposal for a tailored enhanced recovery protocol and preliminary report from a pilot study. Urol Int. 2014; 92:41-49.

11. Schiavina R, et al. MRI Displays the Prostatic Cancer Anatomy and Improves the Bundles Management Before Robot-Assisted Radical Prostatectomy. J Endourol. 2018; 32:315-321.

12. Shafii $M$, et al. is mechanical bowel preparation necessary in patients undergoing cystectomy and urinary diversion? BJU Int. 2002, 89:879-881.

13. Braga $M$, et al. ESPEN Guidelines on Parenteral Nutrition: surgery. Clin Nutr. 2009; 28:378-386.

14. Kouba EJ, Wallen EM, Pruthi RS. Gum chewing stimulates bowel motility in patients undergoing radical cystectomy with urinary diversion. Urology. 2007; 70:1053-1056.

15. Pruthi RS, et al. Fast track program in patients undergoing radical cystectomy: results in 362 consecutive patients. J Am Coll Surg. 2010; 210:93-99.

16. White PF, et al. The role of the anesthesiologist in fast track surgery: from multimedial analgesia to perioperative medical care. Anesth Analg. 2007; 104:1380-1396.

17. Friederich-Freksa $M$, et al. Cystectomy and urinary diversion in the treatment of bladder cancer without artificial respiration. Int Braz J Urol. 2012; 38:645-651.

18. Porreca A, et al. Robot assisted radical cystectomy with totally intracorporeal urinary diversion: initial, single-surgeon's experience after a modified modular training. Minerva Urol Nefrol. 2018; 70:193-201.

19. Saar $M$, et al. Fast-track rehabilitation after robot-assisted laparoscopic cystectomy accelerates postoperative recovery. BJU Int. 2013; 112:E99-E106. 
20. Wilson TG, et al. Best practices in robot-assisted radical cystectomy and urinary reconstruction: recommendations of the Pasadena Consensus Panel, Eur Urol. 2015; 67:363-75.

21. Mineo Bianchi F, et al. Posterior muscle-fascial reconstruction and knotless urethro-neo bladder anastomosis during robot-assisted radical cystectomy: Description of the technique and its impact on urinary continence, Arch Ital Urol Androl. 2019; 91:5-10.

22. Porreca A, et al. Robotic-Assisted Radical Prostatectomy with the Use of Barbed Sutures, Surg Technol Int. 2017; 30:39-43.

23. Porreca A, et al. Bidirectional barbed suture for posterior mus- culofascial reconstruction and knotless vesicourethral anastomosis during robot-assisted radical prostatectomy. Minerva Urol Nefrol. 2018; 70:319-325.

24. Schiavina $R$, et al. Laparoscopic and robotic ureteral stenosis repair: a multi-institutional experience with a long-term follow-up. J Robot Surg. 2016; 10:323-330.

25. Freeks SK, et al. A prospective randomized pilot study evaluating an ERAS protocol versus a standard protocol for patients treated with radical cystectomy and urinary diversion for bladder cancer, World J Urol. 2018; 36:215-220.

\section{Appendix}

\section{Enhanced Recovery Protocol}

Preoperative clinical setting: within 7 days before surgery

- Anestehesiological assessment

- Written dietary recommendations

- Diet with no restrictions

Preoperative days - 24 hours before RC

- Hospital admittance

- Unrestricted clear fluids

- Normal breakfast

- No bowel preparation

Perioperative phase - day of RC

- Clear fluids allowed up to 2 hours before RC

- Nutritional supply 2 hours before surgery (400 mL/200 calories)

- Elastic compressive stockings

- Ceftriaxone 2 g i.v as prophylaxis for infection

Intraoperative phase - day of RC

- Combined general and epidural anesthesia with intrathecal catheter left in place for the first PODs

- Optimized intraoperative intravenous fluid administration

- NGT insertion preoperatively ad removal at the end of surgery

- Reducing intraoperative blood loss

- Antiemetic prophylaxis

- Infiltration of the surgical wound with local anesthetic

Postoperative phase - day of RC

- Ranitidine 150 mg i.v.

- Metoclopramide $25 \mathrm{mg}$ i.v. every 8 hours

- Intravenous analgesia (paracetamol, ketorolac)

- Epidural analgesia (elastomeric pump loaded with naropine)

- Low molecular weight heparin (LMWH) as prophylaxis for thromboembolic events

- Intravenous hydration $(100 \mathrm{~mL} / \mathrm{h})$ of $10 \%$ glucose solution and electrolyte solution

- Mobilization 6 hours after surgery

- Free clear fluids as tolerated

Postoperative phase - POD 1

- Female patients: remove vaginal pack

- Active mobilization

- Respiratory rehabilitation exercises

- 1100 calories diet as tolerated

- Free clear liquids as tolerated

- Analgesia if needed (ropivacaine, paracetamol, ketorolac)

- Metoclopramide 25 mg i.v. every 8 hours

- Ranitidine 150 mg 1 tab/die

- LMWH as prophilaxys

- Chewing gum (1 piece very 2-4 hours), as tolerated

Postoperative phase - POD 2

- 1500 calories diet as tolerated

- Free clear fluids

- Active mobilization

- Drain removal (if drained $<50 \mathrm{~mL} / 24$ hours) 
- Epidural catheter removal

- Neobladder flushes 3 trimes a day (every 8 hours)

- Analgesia if required (paracetamol, ketorolac)

- LMWH as prophylaxis

- Metoclopramide $25 \mathrm{mg}$ i.v. every 8 hours

- Continue ranitidine

Postoperative phase - POD 3

- Active mobilization

- 1650 calories diet

- Analgesia if needed (paracetamol, ketorolac)

- Metoclopramide 25 mg i.v. every 8 hours

- LMWH as prophylaxis

- Continue ranitidine

- Continue neobladder flushing

Postoperative phase - POD 4

- Active mobilization

- 2000 calories diet as tolerated

- Analgesia if needed (paracetamol, ketorolac)

- Metoclopramide 25 mg i.v. every 8 hours

- LMWH as prophylaxis

- Continue ranitidine

- Continue neobladder flushing

Postoperative phase - PODs 5 to 7

- Free diet

- Active mobilization

- If absence of canalization and oralization after 5 days from surgery, start total parenteral nutrition (TPN) and search for any cause

- Continue neobladder flushing

- LMWH as prophylaxis

- Continue ranitidine

Postoperative phase - PODs 8 and 9

- Ureteral stents removal

- Clips removal

- LMWH as prophylaxis

Postoperative phase - PODs 10 and 11

- Schedule for return to home

- LMWH as prophylaxis (up to 18 days after RC)

Postoperative phase - POD 30

- Catheter removal (without neocystogram)

\section{Correspondence}

Daniele Romagnoli, MD (Corresponding Author)

danieleromagnoli87@gmail.com

Marco Giampaoli, MD

Paolo Corsi, MD

Daniele D'Agostino, MD

Angelo Porreca, MD

Robotic Urology and Mini Invasive Urologic Surgery Unit

Abano Terme Hospital, Piazza Cristoforo Colombo 2 - Abano Terme (PD), Italy

Riccardo Schiavina, MD

Lorenzo Bianchi, $M D$

Marco Borghesi, MD

Federico Mineo Bianchi, MD

Andrea Angiolini, MD

Eugenio Brunocilla, MD

Urology Unit, S. Orsola Malpighi University Hospital - Alma Mater Studiorum

Via Pelagio Palagi, 9 Bologna (Italy)

\section{Francesco Chessa}

Carlo Casablanca

St. Orsola Malpighi Universitary Urology Clinic, Bologna (BO), Italy 